\title{
Genome-wide Association Study Identifies Genetic Variants Associated With Early and Sustained Response to (Pegylated) Interferon in Chronic Hepatitis B Patients: The GIANT-B Study
}

\begin{abstract}
Willem P. Brouwer, ${ }^{1, \odot}$ Henry L. Y. Chan, ${ }^{2}$ Pietro Lampertico, ${ }^{3}$ Jinlin Hou, ${ }^{4}$ Pisit Tangkijvanich, ${ }^{5}$ Hendrik W. Reesink, ${ }^{6}$ Wenhong Zhang, ${ }^{7}$ Alessandra Mangia, ${ }^{8}$ Tawesak Tanwandee, ${ }^{9}$ Giuseppe Montalto, ${ }^{10}$ Kris Simon, ${ }^{11}$ Necati Ormeci, ${ }^{12}$ Liang Chen, ${ }^{13}$ Fehmi Tabak, ${ }^{14}$ Fulya Gunsar, ${ }^{15}$ Robert Flisiak, ${ }^{16}$ Peter Ferenci, ${ }^{17}$ Meral Akdogan, ${ }^{18}$ Filiz Akyuz, ${ }^{19}$ Nattiya Hirankarn, ${ }^{5}$ Louis Jansen, ${ }^{6}$ Vincent Wai-Sun Wong, ${ }^{2}$ Roberta Soffredini, ${ }^{3}$ Xieer Liang, ${ }^{4}$ Shalom Chen, Zwier M. A. Groothuismink, ${ }^{1}$ Rosanna Santoro, ${ }^{8}$ Jerzy Jaroszewicz, ${ }^{16,20}$ Resat Ozaras, ${ }^{14}$ Karin Kozbial, ${ }^{17}$ Mayur Brahmania, ${ }^{21}$ Qing Xie, ${ }^{22}$ Watcharasak Chotiyaputta, ${ }^{9}$ Qi Xun, ${ }^{13}$ Monika Pazgan-Simon, ${ }^{11}$ Erkin Oztas, ${ }^{18}$ Elke Verhey, ${ }^{1}$ Noé R. Montanari, ${ }^{1}$ Jian Sun, ${ }^{4}$ Bettina E. Hansen, ${ }^{1}$ Andre Boonstra, ${ }^{1}$ and Harry L. A. Janssen ${ }^{1,21}$; for the GIANT-B Global Consortium ${ }^{a}$

${ }^{1}$ Gastroenterology and Hepatology, Erasmus Medical Center Rotterdam, The Netherlands; ${ }^{2}$ Department of Medicine and Therapeutics, The Chinese University of Hong Kong; ${ }^{3} \mathrm{Centro} \mathrm{di} \mathrm{riferimento} \mathrm{per}$ la diagnosi e lo studio delle malattie del fegato e delle vie biliari "Angela Maria ed Antonio Migliavacca" Center for Liver Disease, Division of Gastroenterology and Hepatology, Fondazione Istituto di Ricovero e Cura a Carattere Scientifico di natura pubblica Cà Granda Ospedale Maggiore Policlinico, University of Milan, Italy; ${ }^{4}$ State Key Laboratory of Organ Failure Research, Guangdong Provincial Key Laboratory of Viral Hepatitis Research, Hepatology Unit and Dept of Infectious Diseases, Nanfang Hospital, Southern Medical University, Guangzhou, China; ${ }^{5}$ Faculty of Medicine, Chulalongkorn University, Bangkok, Thailand; ${ }^{6}$ Academic Medical Centre, Amsterdam, The Netherlands, ${ }^{7}$ Clinical Center Hepatitis, Institute of Biomedical Science, Huashan hospital "Fu Dan University," Shanghai, China; ${ }^{8}$ Istituto di Ricovero e Cura a Carattere Scientifico di natura pubblica Casa Sollievo della Sofferenza, Foggia, Italy, ${ }^{9}$ Division of Gastroenterology, Department of Medicine, Siriraj Hospital, Mahidol University, Bangkok, Thailand; ${ }^{10}$ Biomedical Department of Internal Medicine and Specialties, University of Palermo, Italy; ${ }^{11}$ Wroclaw University of Medicine Department of Infectious Diseases and Hepatology, Poland; ${ }^{12}$ University of Ankara, Medical School, Turkey; ${ }^{13}$ Shanghai Public Health Center "Fu Dan University," China; ${ }^{14}$ Cerrahpasa Medical Faculty, Department of Infectious Diseases, Istanbul, and ${ }^{15}$ Ege University Medical School, Izmir, Turkey; ${ }^{16}$ Department of Infectious Diseases and Hepatology, Medical University of Bialystok, Poland; ${ }^{17}$ Medical University of Vienna, Austria, ${ }^{18}$ Yuksek Ihsitas Hospital, Ankara, and ${ }^{19}$ Istanbul Üniversitesi Istanbul Tip Fakültesi Hastanesi, Istanbul, Turkey; ${ }^{20}$ Department of Infectious Diseases and Hepatology, Medical University of Silesia, Katowice, Poland, ${ }^{21}$ Liver Clinic, Toronto General Hospital, University Health Network, Toronto, Canada; and ${ }^{22}$ Shanghai Ruijin Hospital, Jiao Tong University School of Medicine, Shanghai, China
\end{abstract}

Background. (Pegylated) Interferon ([Peg]IFN) therapy leads to response in a minority of chronic hepatitis B (CHB) patients. Host genetic determinants of response are therefore in demand.

Methods. In this genome-wide association study (GWAS), CHB patients, treated with (Peg)IFN for at least 12 weeks \pm nucleos(t)ide analogues within randomized trials or as standard of care, were recruited at 21 centers from Europe, Asia, and North America. Response at 24 weeks after (Peg)IFN treatment was defined as combined hepatitis B e antigen ( $\mathrm{HBeAg}$ ) loss with hepatitis B virus (HBV) DNA $<2000 \mathrm{IU} / \mathrm{mL}$, or an HBV DNA $<2000 \mathrm{IU} / \mathrm{mL}$ for HBeAg-negative patients.

Results. Of 1144 patients, 1058 (92\%) patients were included in the GWAS analysis. In total, 282 (31\%) patients achieved the response and $4 \%$ hepatitis B surface antigen (HBsAg) loss. GWAS analysis stratified by HBeAg status, adjusted for age, sex, and the 4 ancestry components identified PRELID2 rs371991 $\left(\mathrm{B}=-0.74\right.$, standard error $\left.[\mathrm{SE}]=0.16, P=3.44 \times 10^{-6}\right)$ for $\mathrm{HBeAg}$-positive patients. Importantly, PRELID2 was cross-validated for long-term response in HBeAg-negative patients. G3BP2 rs3821977 (B = 1.13, $\mathrm{SE}=0.24, P=2.46 \times 10^{-6}$ ) was associated with response in HBeAg-negative patients. G3BP2 has a role in the interferon pathway and was further examined in peripheral blood mononuclear cells of healthy controls stimulated with IFNa and TLR8. After stimulation, less production of IP-10 and interleukin (IL)-10 proteins and more production of IL-8 were observed with the G3BP2 G-allele.

Conclusions. Although no genome-wide significant hits were found, the current GWAS identified genetic variants associated with (Peg)IFN response in CHB. The current findings could pave the way for gene polymorphism-guided clinical counseling, both in the setting of (Peg)IFN and the natural history, and possibly for new immune-modulating therapies.

Clinical Trials Registation: NCT01401400.

Keywords. peginterferon; chronic hepatitis B; response; GWAS; genetics.

Received 8 August 2018; editorial decision 15 January 2019; accepted 11 March 2019; published online February 2, 2019.

aGIANT-B global consortium members are listed in the Notes

Correspondence: Harry L.A. Janssen MD, PhD, Francis Family Chair in Liver Research, Director Toronto Centre for Liver Disease, Professor of Medicine, University of Toronto, Toronto General Hospital, 200 Elizabeth Street, Eaton Building 9th floor Room 234 (9 EB 234), Toronto ON M5G 2C4 (Harry.janssen@uhn.ca).

Clinical Infectious Diseases ${ }^{\circledR} \quad 2019 ; X X(X X): 1-11$

(c) The Author(s) 2019. Published by Oxford University Press for the Infectious Diseases Society of America. This is an Open Access article distributed under the terms of the Creative Commons Attribution-NonCommercial-NoDerivs licence (http://creativecommons.org/licenses/ by-nc-nd/4.0/), which permits non-commercial reproduction and distribution of the work, in any medium, provided the original work is not altered or transformed in any way, and that the work is properly cited. For commercial re-use, please contact journals.permissions@oup.com DOI: $10.1093 / \mathrm{cid} / \mathrm{ciz} 084$
Approximately 250 million people worldwide have evidence of a chronic hepatitis $\mathrm{B}(\mathrm{CHB})$ infection, which may progress to liver cirrhosis, liver decompensation, hepatocellular carcinoma (HCC), and death. The aim of antiviral therapy is to improve the quality of life and the long-term prognosis [1-3]. Current internationally recommended treatments Tenofovir, Entecavir, and (pegylated) interferon ([Peg]IFN), which can reduce viral load and hepatic necroinflammation, decrease the risk of HCC and complications of cirrhosis [4-6]. (Peg)IFN has both direct antiviral and immune modulating effects. The main advantages of this agent include a finite course of treatment and the lack 
of drug resistance. It leads to an improved prognosis and high rates of hepatitis B surface antigen (HBsAg) loss during longterm follow-up in those with a favorable response [7]. However, it requires subcutaneous injections and carries considerable side effects. In addition, only $20-30 \%$ of treated patients have a sustained response to treatment [8-11]. It is therefore important to identify host genetic determinants of response to reduce the costs and side effects of treatment and make this treatment modality more acceptable to patients. Genetic host studies on response to (Peg)IFN provide substantial knowledge on the interaction between the host and the virus to induce immune control, both for immune modifying therapy and the natural history of disease. Numerous small studies conducted with selected single-nucleotide polymorphism (SNP) have shown associations with (Peg)IFN response; however, to date, a large genome-wide association study (GWAS) to predict the response to (Peg)IFN in CHB patients has not been performed. Causal or regulating SNPs in genes modifying the immune response can be identified through a GWAS and can be used to assess the chance of response to treatment and select patients who have a high probability of response to (Peg)IFN. This would potentially pave the way for further functional follow-up and clinical validation on gene polymorphisms-guided therapeutic stratification, both in the setting of (Peg)IFN and possibly for new therapeutic agents.

\section{PATIENTS AND METHODS}

\section{Patients}

In this investigator-initiated multicentre global GWA study, CHB patients treated with PegIFN alpha or conventional interferon $\alpha$ (IFN $\alpha$ ) within randomized controlled trials, prospective cohort studies, or as part of standard of care at 21 tertiary care centers from Europe, Asia, and North America were initially eligible for inclusion. The inclusion and exclusion criteria for the trials are described elsewhere [7, 8, 10, 12-22]. For the current study, the minimal duration of therapy was 12 weeks. Patients who received combined treatment of PegIFN and nucleos $(\mathrm{t})$ ide analogues (NA) or ribavirin therapy were also included, because combination treatment with these agents does not influence response rates at 6 months post-treatment as compared to PegIFN alone $[7-11,13,16,17,23]$. PegIFN add-on therapy to short-term [12] or long-term [14, 20-22] NA treatment was also allowed. Patients were excluded in case of a hepatitis $\mathrm{C}$, hepatitis delta, or human immunodeficiency virus coinfection. An overview of the origin of included patients can be found in Supplementary Table 1. The study was conducted in agreement with the guidelines of the Declaration of Helsinki and the principles of Good Clinical Practice. The study was approved by the ethics committee of each participating centre. All patients gave written informed consent according to standards of the local ethics committees at each of the participating centres. All authors had access to the study data and reviewed and approved the final manuscript. The study protocol can be accessed at clinicaltrials.gov, identifier NCT01401400.

\section{Endpoints}

The primary endpoint was assessed at 24 weeks post (Peg) IFN treatment, and the primary response was defined as combined hepatitis B e antigen ( $\mathrm{HBeAg}$ ) loss with a hepatitis B virus (HBV) DNA $<2000 \mathrm{IU} / \mathrm{mL}$ for $\mathrm{HBeAg}$ positive patients, and an HBV DNA level $<2000 \mathrm{IU} / \mathrm{mL}$ for $\mathrm{HBeAg}$-negative patients, including confirmed $\mathrm{HBsAg}$ loss, according to standard definitions reported by clinical practice guidelines [1-3]. Other endpoints considered were sustained response (ie, patients with both response at 24 weeks post-(Peg)IFN and at end of follow-up) and end of follow-up response (ie, includes patients who achieved response during follow-up, but excluding those who lost response). Combination of the primary endpoint with ALT normalization was considered as well and can be found in the Supplementary Materials.

\section{DNA Extraction, Host Genotyping, and Laboratory Measurements}

These data can be found in the Supplementary Materials.

\section{Genome-wide Association and Statistical Analysis}

Data were analyzed according to genetic analysis populations (GAP). Patients included in the GAP comprise all patients in the study who were successfully genotyped (ie, passed the quality control steps) and were treated with (Peg)IFN for at least 12 weeks. In this analysis, if data on outcome were missing, patients were not considered in the analysis. GAP analysis was applied both for the primary as well as the secondary endpoints. For each genotyped or imputed SNP, binary logistic regression analysis of the primary response was performed with additive SNP effects for the total cohort with adjustment for sex, age, baseline $\mathrm{HBeAg}$ status, and 4 ancestry principal components (PCs). These PCs represent the genetic ethnicity, which are extracted from the genetic data. Next to a GWAS for the total cohort, we performed a stratified GWAS by $\mathrm{HBeAg}$ status as well, because patients with $\mathrm{HBeAg}$-negative $\mathrm{CHB}$ may be a more genetically selected subgroup and therefore possibly biologically different compared to those with HBeAg-positive CHB [1-3]. RVtests were used for the GWAS analysis [24]. With RVtests, dosage information was used instead of best-guess genotypes. Moreover, all variants with a minor allele frequency $<1 \%$ were discarded. Finally, with RVtests, the Wald test was used to determine significance [24]. A $P$-value of less than $5 \times 10^{-8}$ was considered genomewide significant. Q-Q plots were generated to inspect the consistency between the resultant and expected test statistics (ie, any evidence of genomic inflation), and genomic control was applied to adjust for any residual inflation. 


\section{Sensitivity Analyses}

We performed a multivariable analysis including the obtained SNPs of interest, additionally adjusted for the duration of (Peg)IFN treatment, HBV DNA load and ALT at baseline. Moreover, a sensitivity analysis for the combination of the primary endpoint with ALT normalization, a stratified analysis by physician-reported ethnicity, and an analysis for the treatment regimen received were performed. Finally, a review of previously described SNPs associated with (PEG)IFN response was performed. All sensitivity analysis can be found in the Supplementary Materials.

\section{RESULTS}

\section{Patient Characteristics}

In total, 1695 patients treated with (Peg)IFN were identified at 21 centers worldwide. The patient selection can be found in the study workflow (Figure 1). After selection procedures, a GWAS attempt could be made on 1144 (67\%) of which 1058 (92\%) were successfully included in the GWAS cohort after quality control checks (Table 1). In sum, 534 (51\%) patients were treated as part of (randomized) trials or per study protocols, and 524 (50\%) patients were treated as part of standard of care [7, 8, 10, 12-22] (Supplementary Materials). In total, 923 patients were analyzed per GAP for the primary endpoint.

\section{Primary Outcome and Follow-up}

Per GAP analysis, 282 (31\%) out of 923 patients achieved the primary response ( $12 \%$ with HBsAg loss, $4 \%$ of the GAP cohort). The primary response rate for white patients was $22 \%$ (60/278), and 34\% (217/636) for Asians. For HBeAg-positive patients the primary response rate was 24\% (121/509) and for HBeAg-negative patients 39\% (161/414). Patients were followed for a median of 146 weeks (interquartile range [IQR] 96-401) from baseline, which was a median of 99 weeks (IQR 48-353) from cessation of (Peg)IFN treatment.

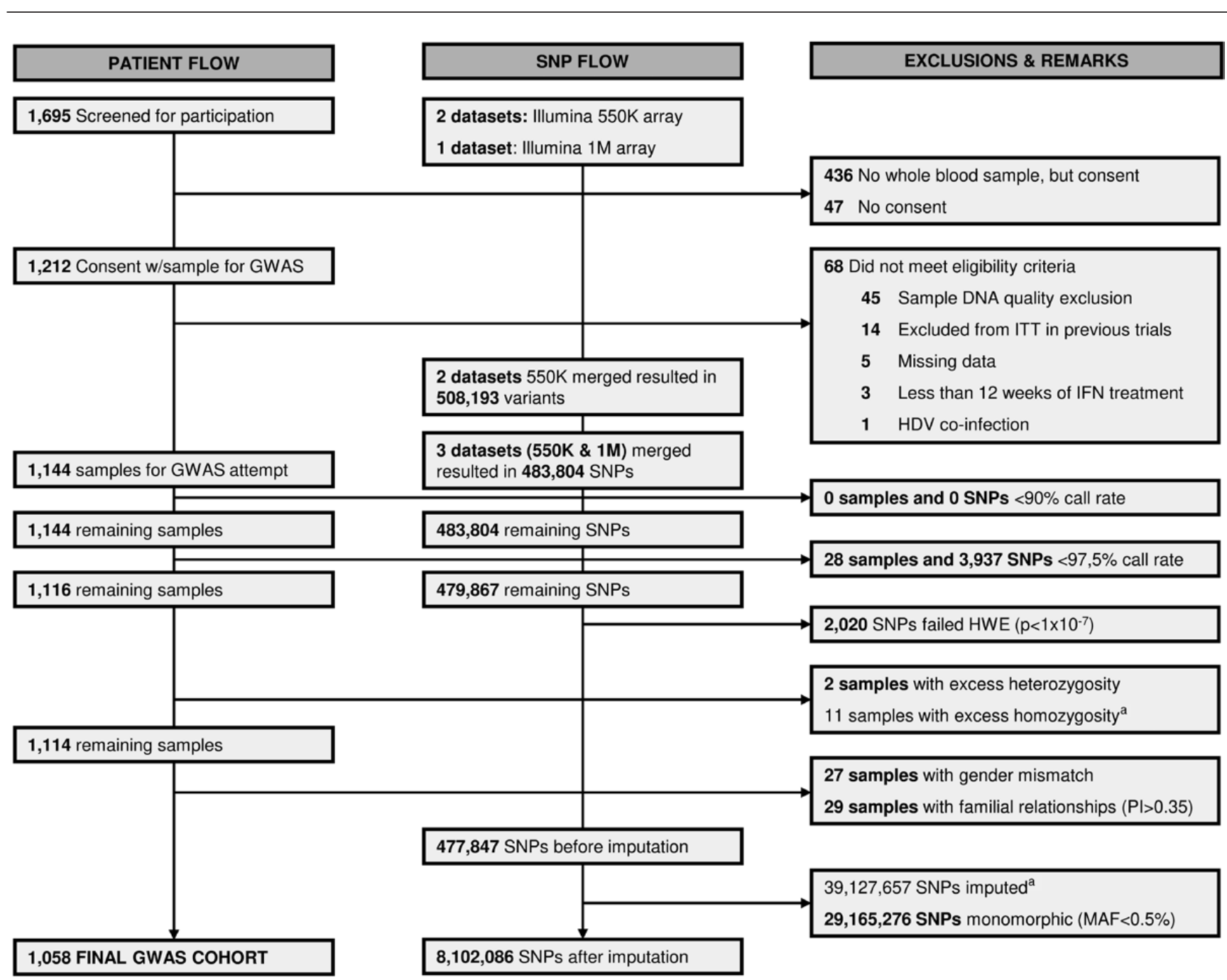

Figure 1. Study workflow for both samples and single-nucleotide polymorphisms. Abbreviations: GWAS, genome-wide association study; HDV, hepatitis Delta virus infection; HWE, Hardy-Weinberg equilibrium; IFN, interferon; ITT, intention-to-treat analysis; MAF, minor allele frequency; PI, PI-HAT, variable calculated by PLINK from the

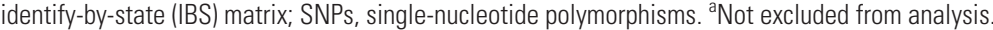




\begin{tabular}{|c|c|c|c|}
\hline Characteristics & All Patients ( $n=1058$ ) & HBeAg-positive ( $N=549$ ) & HBeAg-negative $(\mathrm{N}=509$ ) \\
\hline \multicolumn{4}{|l|}{ Demography } \\
\hline Mean (SD) age, years & $39(11)$ & $35(10)$ & $43(10)$ \\
\hline Male, $n(\%)$ & $753(71 \%)$ & $374(68 \%)$ & $379(75 \%)$ \\
\hline \multicolumn{4}{|l|}{ Reported ethnicity ${ }^{\mathrm{a}}$} \\
\hline Asian & $646(61 \%)$ & $429(78 \%)$ & $217(43 \%)$ \\
\hline White & $375(35 \%)$ & $101(18 \%)$ & $274(54 \%)$ \\
\hline African/other & $37(3 \%)$ & $19(4 \%)$ & $18(3 \%)$ \\
\hline Previous IFN therapy, $\mathrm{n}(\%)$ & $88(8 \%)$ & $36(7 \%)$ & $52(10 \%)$ \\
\hline \multicolumn{4}{|l|}{ Therapy allocation, n (\%) } \\
\hline PeglFN monotherapy & $551(52 \%)$ & $254(46 \%)$ & $297(58 \%)$ \\
\hline PeglFN + NA & $239(23 \%)$ & $140(26 \%)$ & $99(20 \%)$ \\
\hline PegIFN add-on & $90(9 \%)$ & $86(16 \%)$ & $4(1 \%)$ \\
\hline $\mathrm{IFN} \pm \mathrm{NA}$ & $156(15 \%)$ & $69(12 \%)$ & $87(17 \%)$ \\
\hline PeglFN + Ribavirin & $22(2 \%)$ & 0 & $22(4 \%)$ \\
\hline Duration of (Peg)IFN, weeks & $49(18)$ & $44(14)$ & $55(20)$ \\
\hline (Peg)IFN as part of study/trial & $534(51 \%)$ & $308(56 \%)$ & $226(44 \%)$ \\
\hline \multicolumn{4}{|l|}{ Laboratory results, mean (SD) } \\
\hline $\operatorname{ALT}(U / L)^{b}$ & $2.9(3.0)$ & $3.0(2.9)$ & $2.8(3.0)$ \\
\hline HBV DNA, $\log I U / m L$ & $5.7(2.2)$ & $6.4(2.2)$ & $5.0(1.9)$ \\
\hline $\mathrm{HBsAg}, \log \mathrm{IU} / \mathrm{mL}^{\mathrm{c}}$ & $3.5(1.0)$ & $3.7(1.0)$ & $3.2(0.8)$ \\
\hline \multicolumn{4}{|l|}{ HBV genotype, $n(\%)^{d}$} \\
\hline A & $59(6 \%)$ & $35(6 \%)$ & $24(5 \%)$ \\
\hline B & $78(7 \%)$ & $40(7 \%)$ & $38(8 \%)$ \\
\hline C & $196(19 \%)$ & $102(19 \%)$ & $94(19 \%)$ \\
\hline $\mathrm{D}$ & $195(18 \%)$ & $46(8 \%)$ & 149 (29\%) \\
\hline Other & $23(2 \%)$ & $10(2 \%)$ & $13(3 \%)$ \\
\hline Liver cirrhosis & $79(8 \%)$ & $26(5 \%)$ & $53(10 \%)$ \\
\hline \multicolumn{4}{|l|}{ Long-term follow-up } \\
\hline Weeks from baseline, median (IQR) & $146(96-401)$ & $144(77-384)$ & $188(120-418)$ \\
\hline Weeks from EOT, median (IQR) & $99(48-353)$ & $96(32-347)$ & $135(72-366)$ \\
\hline \multicolumn{4}{|l|}{ Primary response GAP, n (\%) } \\
\hline At 24 weeks post IFN & 282/923 (31\%) & $121 / 509(24 \%)$ & $161 / 414(39 \%)$ \\
\hline At end of follow-up & $261 / 1028(25 \%)$ & $115 / 534(22 \%)$ & 146/494 (30\%) \\
\hline Sustained response $\mathrm{e}^{\mathrm{e}}$ & $196 / 902(22 \%)$ & $94 / 497(19 \%)$ & $102 / 405(25 \%)$ \\
\hline
\end{tabular}

Liver cirrhosis was based on pathology or radiology \pm clinical characteristics or the combination of these.

Abbreviations: base, baseline (start (Peg)IFN treatment); EOT, end of treatment; GAP, genetic analysis populations; HBeAg, hepatitis B e antigen; HBV, hepatitis B virus; IFN, interferon; IQR, interquartile range; NA, nucleos(t)ide analogue; SD, standard deviation.

${ }^{a}$ Reported ethnicity by local investigator. This does not resemble the ethnicity based on genetics (ancestry principal components) used in the genome-wide association study (GWAS) analyses.

${ }^{\mathrm{b}}$ Multiples of upper limit of the normal range.

${ }^{\mathrm{c}} 315(30 \%)$ missing.

${ }^{d} 507$ (48\%) missing.

eSustained response was defined as patients with response both at 24 weeks post IFN and at end of follow-up.

\section{Genome-wide Association Analysis}

First, a GWAS was performed for the complete cohort (GAP $\mathrm{n}=923)$ adjusted for sex, age, 4 ancestry principal components, and baseline HBeAg status. The Q-Q plot is shown in Figure 2. There were no genome-wide significant associations, but 3 suggestive loci $\left(P<5.0 \times 10^{-6}\right)$ were found (Table 2 and Supplementary Table 2). Of these, $r s 78900671 \mathrm{GC}$ allele (TRAPPC9, COL22A1) had the strongest association (response of best-guess genotypes GG vs GC $28.4 \%$ vs $59 \%$; additive model $\beta=1.434$, standard error $[\mathrm{SE}]=0.29, P=6.43 \times 10^{-7}$ ). Figure 3 shows the Manhattan plot; for the suggestive loci, zoomed-in versions of the Manhattan plot are illustrated in the
Supplementary Materials. Moreover, both a sensitivity analysis and a look-up of previously described SNPs can be found in the Supplementary Materials as well.

\section{Genome-wide Association Analysis Stratified by Baseline HBeAg Status} A subgroup specific GWAS was performed stratified by HBeAg status with adjustment for sex, age, and 4 ancestry principal components. For both groups there were no genome-wide significant associations, but suggestive loci were identified $\left(P<5.0 \times 10^{-6}\right)$. The Q-Q and Manhattan plots are shown in Figures 2 and 3.

For HBeAg-positive patients, rs371991 on chromosome 5 (PRELID2) was associated with the primary outcome (additive 
A

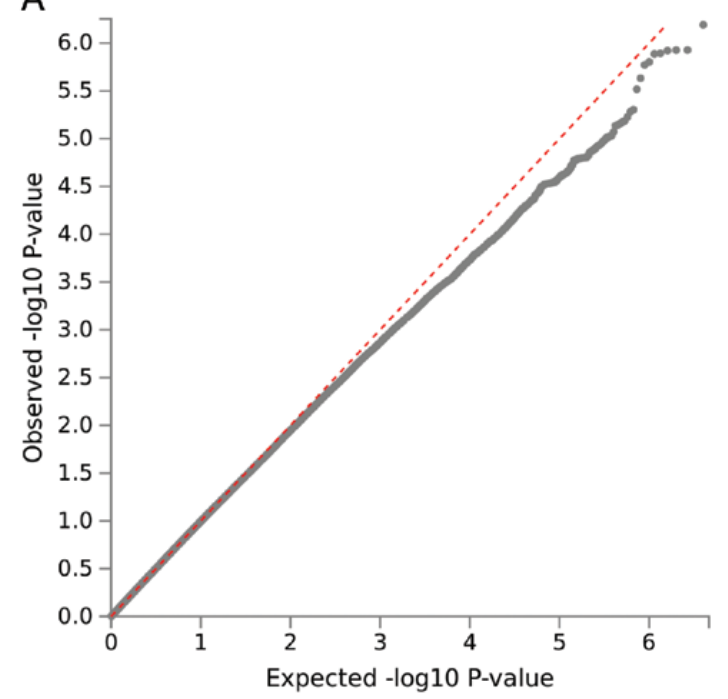

B

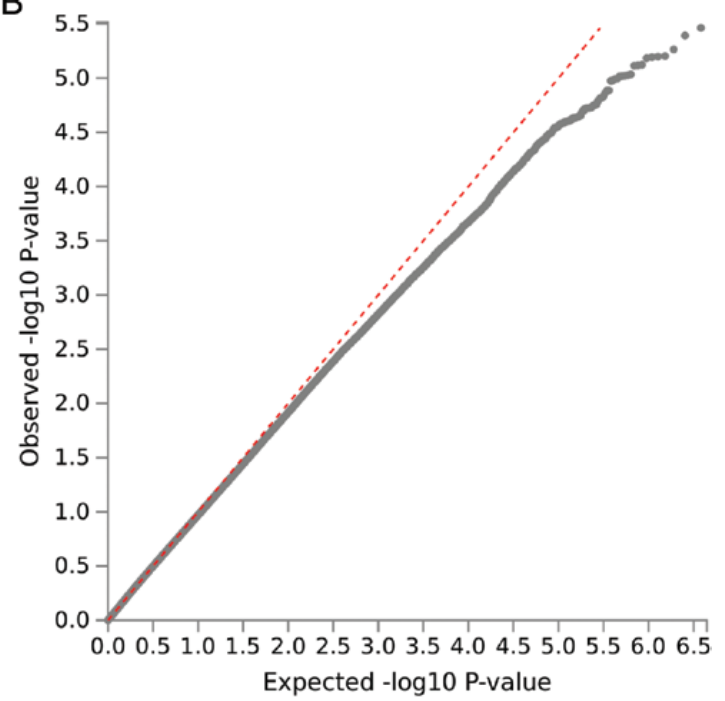

C

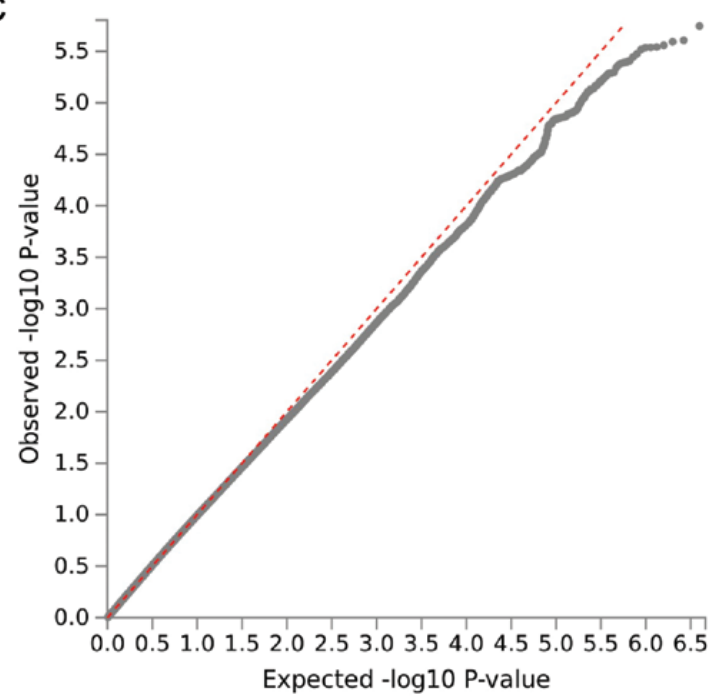

Figure 2. Q- 0 plots for the complete cohort $(A)$, HBeAg-positive patients (B) and HBeAg-negative patients (C). Abbreviation: HBeAg, hepatitis B e antigen.

$\beta=-0.74, \mathrm{SE}=0.16, P=3.44 \times 10^{-6}$, Table 2). Response rates for best-guess genotypes were $38 \%, 23 \%$, and $13 \%$ for $\mathrm{AA}, \mathrm{AG}$, and GG, respectively (Figure 4). As a sensitivity analysis, a multivariable analysis additionally adjusted for the duration of (Peg) IFN treatment, HBV DNA load, and ALT at baseline, PRELID2 remained associated with the primary response (additive $\beta=-0.67, \mathrm{SE}=0.16$, odds ratio $[\mathrm{OR}]=0.51, P=3.70 \times 10^{-5}$ ). PRELID2 was also associated with response at end of follow-up $(P<.001$, Figure 4$)$, with sustained response $(P=.001$, Figure 4$)$, and with combined HBeAg loss with HBV DNA $<2000 \mathrm{IU} / \mathrm{mL}$ and ALT normalization $(P<.001$, Supplementary Materials) for HBeAg-positive patients.

For HBeAg-negative patients the most interesting top-hit SNP associated with the primary response was $r s 3821977(\beta=1.13$, $\mathrm{SE}=0.24, P=2.46 \times 10^{-6}$ ), which is located on chromosome 4 , intronic within gene G3BP2, which has a function within the IFN pathway [25]. Response rates for best-guess genotypes were $15 \%, 22 \%$, and $49 \%$ for AA, AG, and GG, respectively (Figure 4). In sensitivity analysis, this association remained strong after adjustment by the aforementioned variables. G3BP2 was also associated with sustained response, response at long-term follow-up (Figure 4) and with combined HBV DNA $<2000 \mathrm{IU} / \mathrm{ml}$ with ALT normalization $(P<.001$, Supplementary Materials).

\section{PRELID2 Cross-validation in HBeAg-negative Patients}

The association of PRELID2 rs371991 with response in HBeAgpositive patients was further examined in $\mathrm{HBeAg}$-negative patients. Here, we independently validated this association for HBeAg-negative patients at long-term follow-up (sustained response: AA vs AG vs GG: $32 \%, 26 \%, 19 \%$; response at end of 
follow-up: $38 \%, 31 \%, 23 \%, P=.031$ and $P=.008$, respectively, Figure 4). The association of G3BP2 with response in HBeAgnegative patients could not be validated upstream the natural history in HBeAg-positive patients $(P>.05)$.

G3BP2 Gene Function Shows a Differential Effect on IL-8, IP-10, and IL-10 Protein Levels

Because G3BP2 rs3821977 is described as a factor within the IFN response pathway [25], we further focused on the consequence of the specific SNP genotype on the functionality of blood leukocytes (cytokine production of predominantly monocytes and plasmacytoid dendritic cells) in response to exposure to IFN in healthy controls. The methods for this analysis can be found in the Supplementary Materials. In short, we genotyped healthy controls for the SNP and stimulated peripheral blood mononuclear cells (PBMCs) with IFN alone or in combination with R848 (TLR7/8 ligand). Interleukin (IL)-8, IP-10, and IL-10 showed different trends between groups upon IFN+TLR7/8 stimulation. Although IL-8 was higher in the GG than the AA group, IP-10 and IL-10 exhibited higher levels in the AA group (Figure 5). For a subset of patients from the GWAS cohort, baseline IP-10 levels were available, which showed no differences across the G3BP2 genotypes pretreatment $(P>.5)$. The results for the other cytokines and in relation to medium or IFNa alone, can be found in the Supplementary Materials.

\section{DISCUSSION}

In the current global investigator-initiated GWAS, we studied the largest (Peg)IFN treated CHB cohort to date, and identified TRAPPC9, PRELID2 and G3BP2 to be associated with both short and long-term response to (Peg)IFN. Although not genome-wide significant, these top-hit SNPs were suggestive of a meaningful association $\left(P<10^{-6}\right)$, across all ethnicities. Moreover, these SNPs were associated with, or were found within, different immune response pathways. If further corroborated, this may have important implications for both the natural history of CHB and for current PegIFN therapy that should be individualized to patients with the highest likelihood of response. Furthermore, PegIFN is frequently used as an agent to decrease cccDNA and modify the immune response in studies on combination treatment, aiming for functional cure. The outcome of this study may also help us to learn about the biology and likelihood of response to the many other new compounds in development to reach functional cure of $\mathrm{CHB}$.

We performed in a large number of patients 3 GWAS: 1 for the complete group of $\mathrm{CHB}$ patients adjusted for $\mathrm{HBeAg}$ status, and also 2 separate GWAS stratified by HBeAg. For HBeAg-positive patients, the main finding was the association of PRELID2 with (Peg)IFN response. This was observed for response at 24 weeks off-treatment, as well as sustained and long-term follow-up response. Further verifying this association, PRELID2 also showed an association with 
A

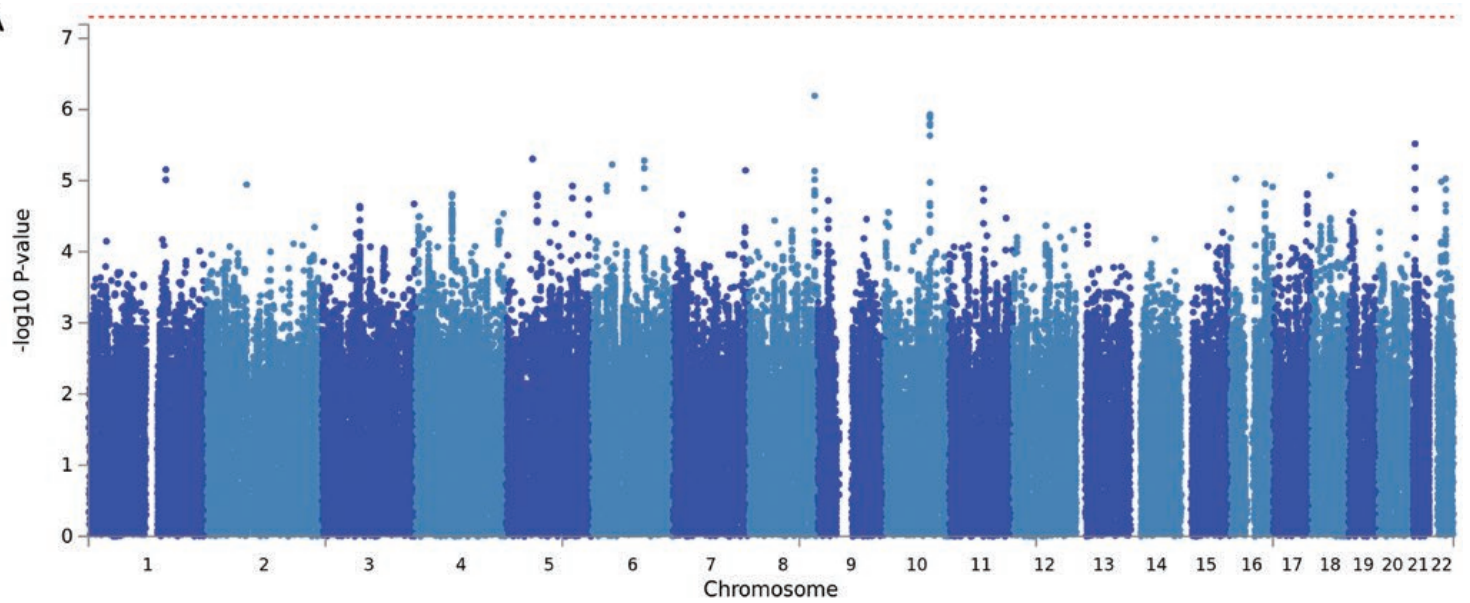

B

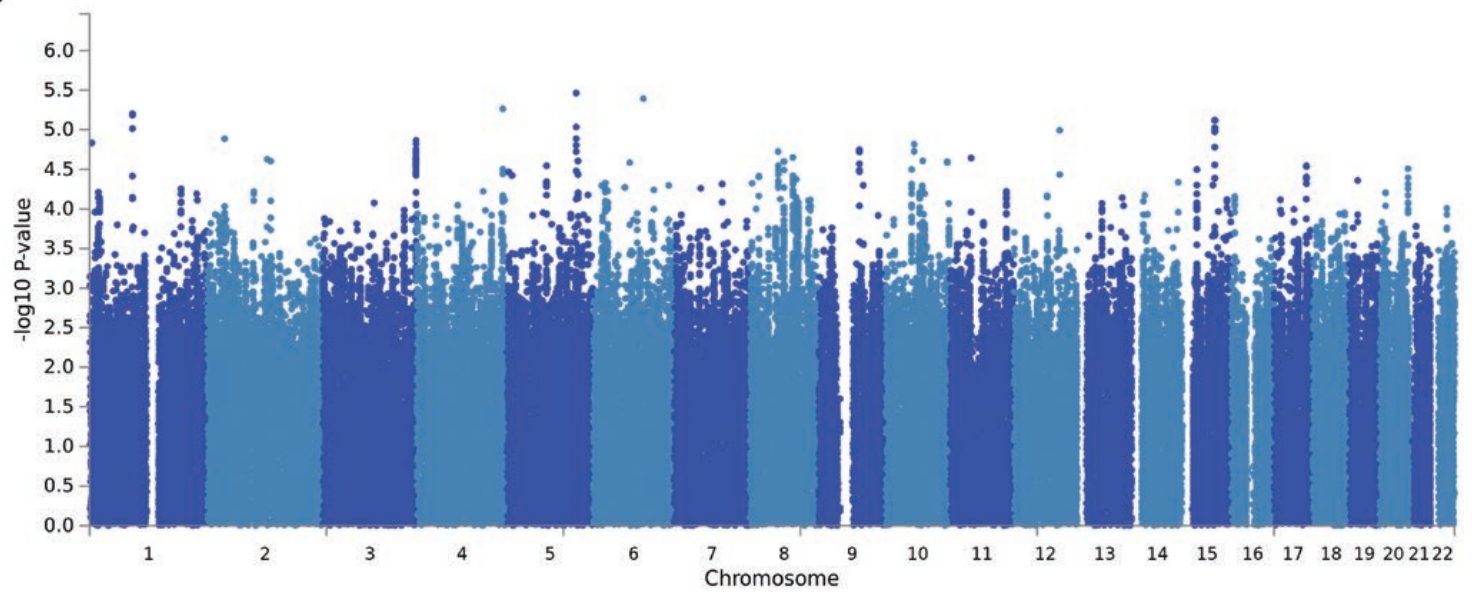

C

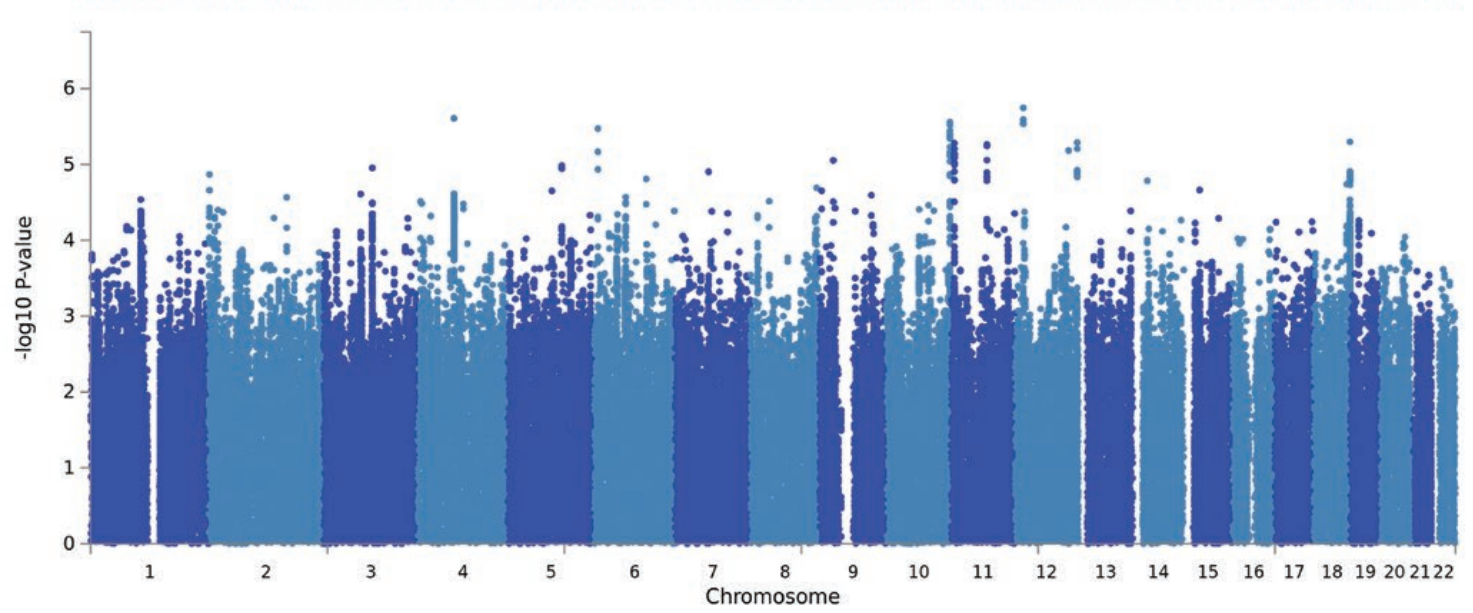

Figure 3. Manhattan plots for the genome-wide association study for the complete cohort $(A)$, HBeAg-positive patients, $(B)$ and $\mathrm{HBeAg-negative} \mathrm{patients}(C)$. Abbreviation: $\mathrm{HBeAg}$, hepatitis B e antigen.

sustained response in an independent cross-validation analysis of HBeAg-negative patients, suggesting this effect is preserved along the natural history of the disease. PRELID2 is known to downregulate STAT6. Located downstream the IL-4 receptor, lower levels of STAT6 may impair T-helper cell differentiation and T-cell survival, which are known to be required processes for an effective HBV-specific T-cell response $[26,27]$. This suggests a potential role for PRELID2 in CHB, likely via attenuating T-cell activity. To our knowledge, no other information is currently available on the involvement of PRELID2 in immune-mediated diseases. 
Another important finding of the current study that came from the GWAS in HBeAg-negative patients, were we found G3BP2 to have the strongest association with both short-term and long-term (Peg)IFN response. This gene has previously been associated with the IFN signaling response as antiviral mediator in the context of viral infection [25]. Therefore, we evaluated whether the SNP could recapitulate the observed association at the functional level. Overnight stimulation of PBMC of healthy controls showed lower protein levels of IL-10 and IP-10, and higher levels of IL-8 with the G3BP2 response allele G. IL-10 is a well-known immunoregulatory cytokine, which can downregulate T-cell immunity and has a significant role in viral resolution or persistence [26]. Lower levels of IL-10 observed with the G3BP2 G-allele could therefore be associated with a stronger IFN response. In contrast, earlier studies have shown a negative regulation by IL- 8 on the IFNa antiviral response in an HCV system [28, 29], and it is therefore tempting to extrapolate these findings by suggesting that IL- 8 may also influence IP-10 levels.
For the complete cohort, we found rs78900671, within gene COL22A1 and close to TRAPPC9, to have the strongest association with response. TRAPPC9, also known as NIK and IKK $\beta$ binding protein (NIBP), functions as an enhancer of tumor necrosis factor $\alpha$ induced NFkB activation. NFkB is a multipotent transcription factor, which is involved in many biological processes in (innate) immunity, inflammation and apoptosis [30]. Because it has a broad effect on multiple pathways, studies to decipher the functional consequences of the SNP genotype are difficult to perform and fall outside the scope of the current article. This should be further investigated in future studies.

The current study has been a long-term global investigator-initiated effort in which we have managed to gather the largest (Peg)IFN treated CHB cohort to date. Despite this fact, the Q-Q plots suggest that the study may be underpowered, and the genome-wide significance threshold could not be achieved. Nevertheless, we have found interesting genetic regions for further investigation. In addition, we have performed a power analysis prior to the study and aimed for at least 1000 patients

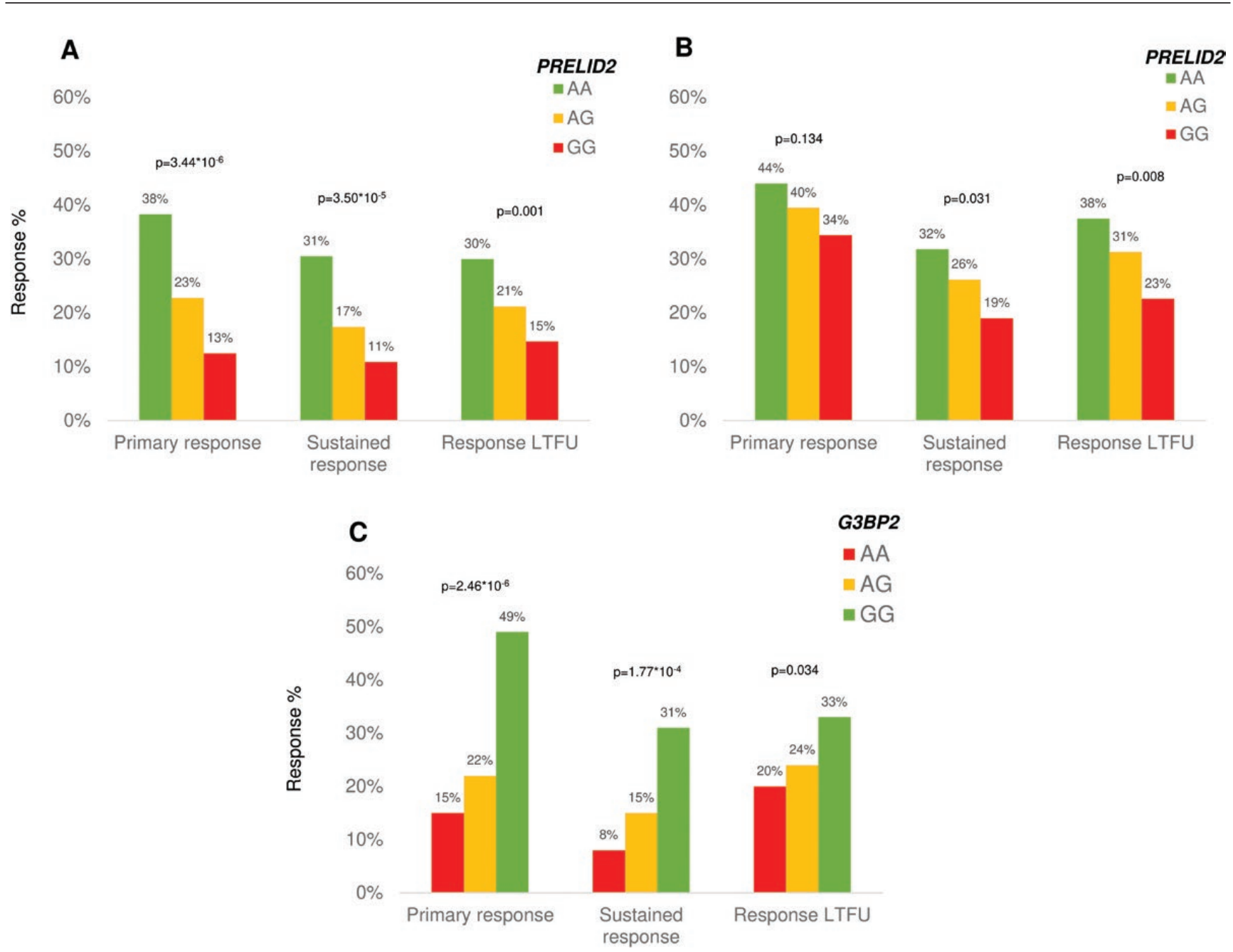

Figure 4. PRELID2 and response for HBeAg-positive $(A)$ and $\mathrm{HBeAg}$-negative patients $(B)$, and G3BP2 and response for $\mathrm{HBeAg-negative} \mathrm{patients} \mathrm{(C).} \mathrm{Abbreviation:} \mathrm{HBeAg}$, hepatitis B e antigen; LTFU, long-term follow-up. 

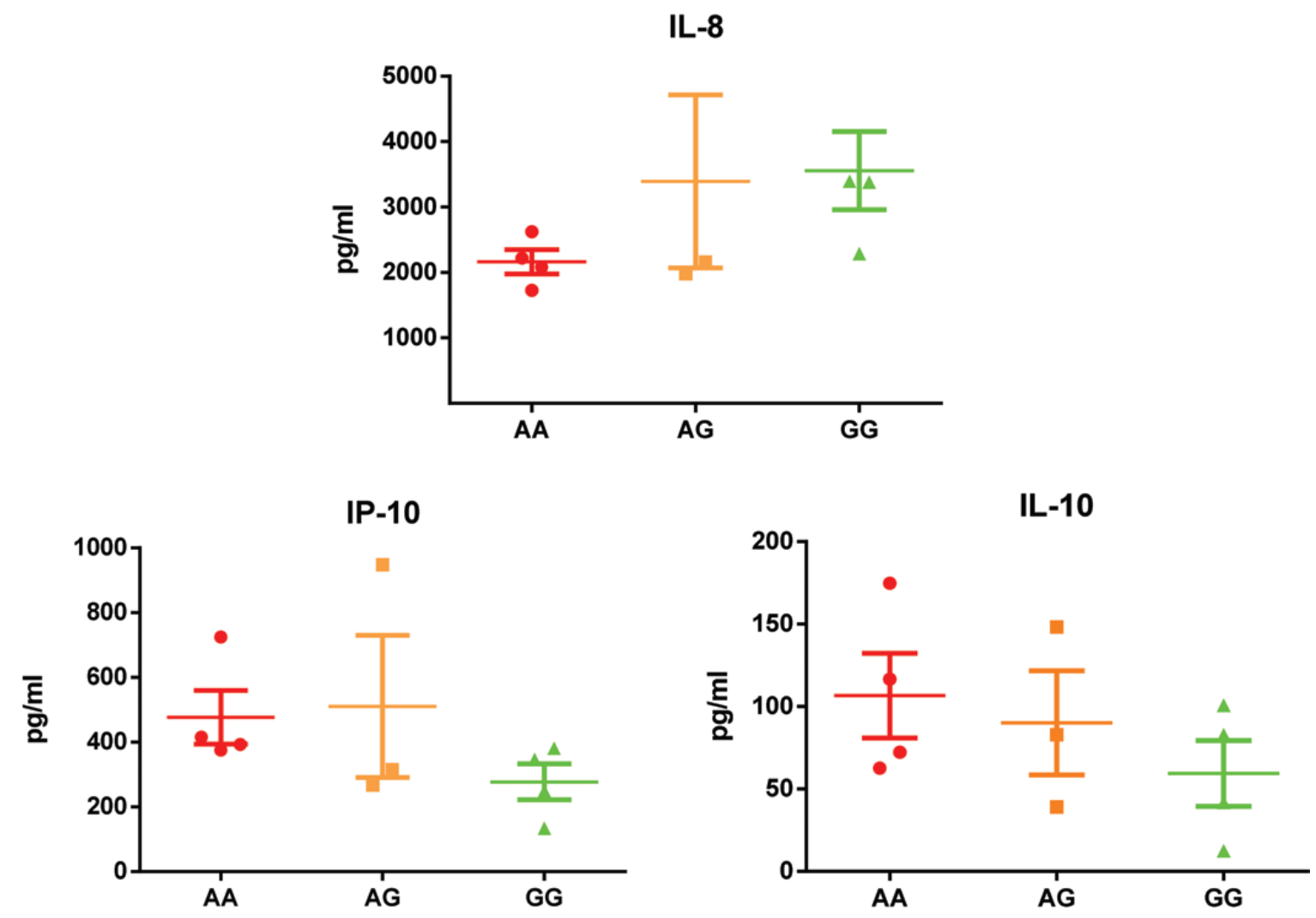

Figure 5. Differential analytes detection for G3BP2 from supernatants of overnight stimulated PBMC with IFNa+R848 (TLR8 ligand). Abbreviations: IL, interleukin; IP, inducible protein; PBMC, peripheral blood mononuclear cell.

(see Supplementary Materials). However, because a GWAS is a genetic exploration and the magnitude of minor allele frequencies and the effect of the gene cannot be predicted, we eventually included as many patients as possible.

Given the retrospective nature of our study, inevitably there is a potential of uncontrolled bias due to patient selection. One bias may be to include relatively more nonresponders because patients who achieve a durable response may be discharged and lost to follow-up. This was not the case for the current study. Patients not included in the GWAS $(\mathrm{N}=637)$ were more often antiviral therapy experienced, collectively had a lower primary response rate ( $22 \%$, vs $31 \%$ in the GWAS cohort, respectively), and had a similar response rate at end of follow-up of $25 \%$. These percentages are in line with previous (Peg)IFN studies. Another potential caveat could be the heterogeneous population, which could drive the findings in a GWAS if not controlled for. To overcome this potential bias, our GWAS analysis was adjusted for the genetic ancestry principal components of the different populations in the analysis. Moreover, stratified by physician-reported ethnicity the associations remained comparable (also see Supplementary Materials). This means that the findings from the current study are important for all reported ethnicities. For $30 \%$ of patients, we did not have information on
HBsAg levels and for $50 \%$ not on HBV genotype, which are both important factors associated with response [23,31-33]. Indeed, HBV genotype is also strongly related to ethnicity. Because we controlled for the genetic population stratification, chances of spurious genetic associations due to HBV genotypic differences driven by a possible overrepresentation of patients with a certain HBV genotype are highly unlikely but cannot be ruled out.

It is imperative to replicate findings discovered by a GWAS. For the current study we were able to cross-validate SNPs identified by the stratified GWAS. Importantly, here we independently cross-validated PRELID2, found in HBeAg-positive patients, for an association with long-term (PEG)IFN response in HBeAg-negative patients. Not unexpectedly, we were not able to replicate G3BP2 in HBeAg-positive patients, because this was a significant SNP for HBeAg-negative patients. This is a genetically more selected subgroup, and it may not be possible to validate upstream the natural history of CHB. Therefore, we investigated the effect of the SNP on IFN responsiveness in healthy controls. Here we showed that G3BP2 differentially affects IP-10, IL-10, and IL-8 protein expression. A potential influence of a CHB infection on the effect of the SNP in response to IFN could not be further investigated in the current study. The reason for this is that $\mathrm{CHB}$ patients in our clinics are 
very heterogeneous in terms of age, phase of infection, antiviral therapy use, and severity of liver disease. Moreover, there is a rather high frequency of Asian patients with a lower frequency of the G3BP2 AA SNP. All of these factors likely affect the in vitro results and may mask the effect of the SNP on the responsiveness to IFN. Therefore, further studies investigating the function of G3BP2 should be performed in $\mathrm{CHB}$ patients.

In conclusion, we performed to our knowledge the first and largest GWAS study on (Peg)IFN treated CHB patients to date. We found genetic variations associated with response for $\mathrm{HBeAg}$-positive and $\mathrm{HBeAg}$-negative patients, irrespective of ethnicity, both when combined as well as in a stratified analysis, and were able to further independently cross-validate these findings. If these results are further confirmed, this may have important clinical implications for further clinical guidance of patients both in the setting of the natural history, as well as for current or innovative immune modulating therapies.

\section{Supplementary Data}

Supplementary materials are available at Clinical Infectious Diseases online. Consisting of data provided by the authors to benefit the reader, the posted materials are not copyedited and are the sole responsibility of the authors, so questions or comments should be addressed to the corresponding author.

\section{Notes}

Acknowledgments. Financial support was given by Merck Sharpe \& Dohme (MSD) Asia, and by the Virgo consortium, funded by the Dutch government project number FES0908, and by the Netherlands Genomics Initiative project number $050-060-452$. The sponsor of this study was the Rotterdam Foundation for Liver Research. The authors would also like to acknowledge the clinical research bureau, Erasmus Medical Center, and all team members from the participating centers.

GIANT-B global consortium contributing investigators: Erasmus MC Rotterdam, The Netherlands: Willem Pieter Brouwer, Bettina Hansen, Elke Verhey, Andre Boonstra, Harry Janssen, Heng Chi, Milan Sonneveld, Noe Rico Montanari, Rob de Knegt. The Chinese University of Hong Kong: Henry Chan, Vincent Wong, Grace Wong. University of Milan, Italy: Pietro Lampertico, Marta Borghi, Alessandro Loglio, Roberta Soffredini. Nanfang Hospital Guangzhou China: Jinlin Hou, Jian Sun, Xieer Liang. Chulalongkorn University, Bangkok, Thailand: Pisit Tangkijvanich, Nattiya Hirankarn, Pimpayao Sodsai, Natthaya Chuaypen. Academic Medical Centre Amsterdam, The Netherlands: Henk Reesink, Louis Jansen. Fu Dan University Shanghai, China: Wenhong Zhang, Shalom Chen. Casa Sollievo della Sofferenza, Foggia, Italy: Alessandra Mangia, Rosanna Santoro. Mahidol University, Bangkok, Thailand: Tawesak Tanwandee, Watcharasak Chotiyaputta. University of Palermo, Palermo, Italy: Guiseppe Montalto. Wroclaw University of Medicine, Wroclaw, Poland: Kris Simon, Monika Pazgan-Simon. University of Ankara, Medical School, Ankara, Turkey: Necati Ormeci. Shanghai Public Health Center: Liang Chen, Xi Qun. Cerrahpasa Medical Faculty, Istanbul, Turkey: Fehmi Tabak, Resat Ozaras; Ege University Medical School, Izmir, Turkey. Fulya Gunsar; Medical University of Bialystok, Poland: Robert Flisiak, Jerzy Jaroszewicz. Medical University of Vienna, Vienna, Austria: Peter Ferenci, Karin Kozbial. Yuksek Ihsitas Hospital, Ankara, Turkey: Meral Akdogan, Erkin Oztas. Istanbul Üniversitesi, Istanbul, Turkey: Filiz Akyuz. Liver Clinic, Toronto General Hospital, Toronto, Canada: Harry Janssen, Mayur Brahmania, Jordan Feld, Seham Noureldin. Shanghai Ruijin Hospital: Simin Guo, Qing Xie.

Author contributions. Study coordination and design, data collection, statistical analysis, writing of manuscript, approval of final version: W. P. B., B. E. H., A. B. Study coordination and design, data collection, critical review of the manuscript, approval of final version: H. L. Y. C., H. L. A. J., J. L. H., P. L. Data collection, critical review of the manuscript, approval of final version: All authors. W. P. B., A. B., H. L. A. J., and B. E. H. had full access to all of the data in the study and take responsibility for the integrity of the data and the accuracy of the data analysis.

Disclaimer. The funding sources did not have any influence on study design, data collection, analysis and interpretation of the data, writing of the report nor the decision to submit for publication.

Financial support. Financial support for this study was provided by Merck Sharpe \& Dohme (MSD) Asia, the Foundation for Liver and Gastrointestinal Research (SLO) in Rotterdam, The Netherlands, and by the Virgo consortium, funded by the Dutch government project number FES0908, and by the Netherlands Genomics Initiative project number 050-060-452. Financial support for the original (randomized) trials are described elsewhere [7, 8, 10, 12-20, 22].

Potential conflicts of interest. H. L.Y. C. is a consultant for AbbVie, Altimmune, Arbutus, Aptorum, Bristol Myers Squibb, Contravir, Gilead Sciences, GRAIL, Intellia, Janssen, MedImmune, Roche, and VIR Biotechnology; and is a speaker for AbbVie, Gilead Sciences, and Roche. P. L. reports speakers' bureau and or advisory board of Bristol-Myers Squibb, Roche, Gilead, GlaxoSmithKline (GSK), MSD, Abbvie, Janssen. H. L.A. J. received grants from and is a consultant for: Bristol Myers Squibb, Gilead Sciences, Novartis, Roche and Merck. A. B. received grants from Roche, Gilead Sciences, Fujirebio, and Janssen and is a consultant for Gilead Sciences. J. H. is a consultant for Abb Vie, Arbutus, Bristol Myers Squibb, Gilead Sciences, Johnson \& Johnson, Roche, and received grants from Bristol Myers Squibb, GSK and Johnson \& Johnson. P. F. is a consultant for Roche, Abbvie, BMS, Gilead, MSD. V. W.-S. W. has served as a consultant for AbbVie, Allergan, Center for Outcomes Research in Liver Diseases, Gilead Sciences, Janssen, Perspectum Diagnostics, Pfizer and TARGET-NASH; and a speaker for Bristol-Myers Squibb, Echosens, Gilead Sciences, and Merck. J. J. is a consultant for AbbVie, BMS, Gilead, and received grants form AbbVie, BMS, Gilead, MSD, and Roche. J. S. reports personal fees from Assembly Bioscience and Gilead. M. P.-S. reports payments from Gilead, Allergan, Beigene, Elsai, Gilead, Abviee, Egora, Alfa Sigma, and Bayer. Q. X. reports payments from Roche, Bristol Meyers Squibb, Novartis, and MSD. H. W. R. reports grants and personal fees from Abbvie, Alnylam, Springer Healthcare, Bristol Myers Squibb, Boehringer Ingelheim, ENYO, Gilead Sciences, Janssen-Cilag, Merck/ MSD, PRA Health Sciences, Regulus, Replicor, Roche, R-Pharm, and Virology Education, outside the submitted work. K. S. has acted as an advisor and lecturer for Abbvie, Gilead, BMS, MSD, Janssen, Alfa-Wasserman, Baxter, Bayer, and Roche-Polska, and has received research support from EISAI, Tobira, Allergan, Intercept, Takeda, Pfizer, and BGB. All other authors report no potential conflicts. All authors have submitted the ICMJE Form for Disclosure of Potential Conflicts of Interest. Conflicts that the editors consider relevant to the content of the manuscript have been disclosed.

\section{References}

1. Sarin SK, Kumar M, Lau GK, et al. Asian-Pacific clinical practice guidelines on the management of hepatitis B: a 2015 update. Hepatol Int 2016; 10:1-98.

2. Terrault NA, Bzowej NH, Chang KM, Hwang JP, Jonas MM, Murad MH; American Association for the Study of Liver Diseases. AASLD guidelines for treatment of chronic hepatitis B. Hepatology 2016; 63:261-83.

3. European Association for the Study of the Liver. EASL 2017 clinical practice guidelines on the management of hepatitis B virus infection. J Hepatol 2017; 67:370-98.

4. Wong VW, Wong GL, Chim AM, et al. Surrogate end points and long-term outcome in patients with chronic hepatitis B. Clin Gastroenterol Hepatol 2009; 7:1113-20

5. Sung JJ, Tsoi KK, Wong VW, Li KC, Chan HL. Meta-analysis: treatment of hepatitis B infection reduces risk of hepatocellular carcinoma. Aliment Pharmacol Ther 2008; 28:1067-77.

6. Liaw YF, Sung JJ, Chow WC, et al; Cirrhosis Asian Lamivudine Multicentre Study Group. Lamivudine for patients with chronic hepatitis B and advanced liver disease. N Engl J Med 2004; 351:1521-31.

7. Buster EH, Flink HJ, Cakaloglu Y, et al. Sustained HBeAg and HBsAg loss after long-term follow-up of $\mathrm{HBeAg}$-positive patients treated with peginterferon alpha-2b. Gastroenterology 2008; 135:459-67.

8. Janssen HL, van Zonneveld M, Senturk H, et al; HBV 99-01 Study Group; Rotterdam Foundation for Liver Research. Pegylated interferon alfa-2b alone or in combination with lamivudine for $\mathrm{HBeAg-positive} \mathrm{chronic} \mathrm{hepatitis} \mathrm{B:} \mathrm{a} \mathrm{ran-}$ domised trial. Lancet 2005; 365:123-9. 
9. Marcellin P, Lau GK, Bonino F, et al; Peginterferon Alfa-2a HBeAg-Negative Chronic Hepatitis B Study Group. Peginterferon alfa-2a alone, lamivudine alone, and the two in combination in patients with $\mathrm{HBeAg-negative} \mathrm{chronic} \mathrm{hepatitis} \mathrm{B}$. N Engl J Med 2004; 351:1206-17.

10. Chan HL, Leung NW, Hui AY, et al. A randomized, controlled trial of combination therapy for chronic hepatitis B: comparing pegylated interferon-alpha2b and lamivudine with lamivudine alone. Ann Intern Med 2005; 142:240-50.

11. Lau GK, Piratvisuth T, Luo KX, et al; Peginterferon Alfa-2a HBeAg-Positive Chronic Hepatitis B Study Group. Peginterferon Alfa-2a, lamivudine, and the combination for HBeAg-positive chronic hepatitis B. N Engl J Med 2005 352:2682-95.

12. Brouwer WP, Xie Q, Sonneveld MJ, et al; ARES Study Group. Adding pegylated interferon to entecavir for hepatitis $B$ e antigen-positive chronic hepatitis B: a multicenter randomized trial (ARES study). Hepatology 2015 61:1512-22.

13. Chan HL, Wong VW, Chim AM, et al. Virological response to different combination regimes of peginterferon alpha- $2 \mathrm{~b}$ and lamivudine in hepatitis $\mathrm{B}$ e antigen positive chronic hepatitis B. Antivir Ther 2007; 12:815-23.

14. Li GJ, Yu YQ, Chen SL, et al. Sequential combination therapy with pegylated interferon leads to loss of hepatitis B surface antigen and hepatitis B e antigen (HBeAg) seroconversion in HBeAg-positive chronic hepatitis B patients receiving long-term entecavir treatment. Antimicrob Agents Chemother 2015; 59:4121-8.

15. Rijckborst V, Ferenci P, Akdogan M, et al; PARC Study Group. Long-term follow-up of hepatitis $\mathrm{B}$ e antigen-negative patients treated with peginterferon a-2a: progressive decrease in hepatitis B surface antigen in responders. Eur J Gastroenterol Hepatol 2012; 24:1012-9.

16. Rijckborst V, ter Borg MJ, Cakaloglu Y, et al; PARC Study Group. A randomized trial of peginterferon alpha-2a with or without ribavirin for HBeAg-negative chronic hepatitis B. Am J Gastroenterol 2010; 105:1762-9.

17. Sprengers D, van der Molen RG, Binda R, et al. In vivo immunization in combination with peg-interferon for chronic hepatitis B virus infection. J Viral Hepat 2007; 14:743-9.

18. Takkenberg RB, Jansen L, de Niet A, et al. Baseline hepatitis B surface antigen (HBsAg) as predictor of sustained $\mathrm{HBsAg}$ loss in chronic hepatitis B patients treated with pegylated interferon- $\alpha 2 a$ and adefovir. Antivir Ther 2013 18:895-904.

19. van Zonneveld M, Honkoop P, Hansen BE, et al. Long-term follow-up of alpha-interferon treatment of patients with chronic hepatitis B. Hepatology 2004 39:804-10.

20. Chi H, Hansen BE, Guo S, et al. Pegylated interferon Alfa-2b add-on treatment in hepatitis B virus envelope antigen-positive chronic hepatitis B patients treated with nucleos(t)ide analogue: a randomized, controlled trial (PEGON). J Infect Dis 2017; 215:1085-93.

21. Janssen HL. PEG-interferon Alfa-2a add-on study in HBeAg negative chronic hepatitis B patients (PAS study). ClinicalTrials.gov NCT01373684.

22. Tangkijvanich $\mathrm{P}$, Chittmittraprap S, Poovorawan K, et al. A randomized clinical trial of peginterferon alpha- $2 \mathrm{~b}$ with or without entecavir in patients with $\mathrm{HBeAg}$ negative chronic hepatitis $\mathrm{B}$ : role of host and viral factors associated with treatment response. J Viral Hepat 2016; 23:427-38.

23. Bonino F, Marcellin P, Lau GK, et al; Peginterferon Alfa-2a HBeAg-Negative Chronic Hepatitis B Study Group. Predicting response to peginterferon alpha-2a, lamivudine and the two combined for HBeAg-negative chronic hepatitis B. Gut 2007; 56:699-705.

24. Zhan X, Hu Y, Li B, Abecasis GR, Liu DJ. RVTESTS: an efficient and comprehensive tool for rare variant association analysis using sequence data. Bioinformatics 2016; 32:1423-6.

25. Bidet K, Dadlani D, Garcia-Blanco MA. G3BP1, G3BP2, and CAPRIN1 are required for translation of interferon stimulated mRNAs and are targeted by a dengue virus non-coding RNA. PLoS Pathog 2014; 10:e1004242.

26. Maini MK, Pallett LJ. Defective T-cell immunity in hepatitis B virus infection: why therapeutic vaccination needs a helping hand. Lancet Gastroenterol Hepatol 2018; 3:192-202.

27. Boeijen LL, Hoogeveen RC, Boonstra A, Lauer GM. Hepatitis B virus infection and the immune response: the big questions. Best Pract Res Clin Gastroenterol 2017; 31:265-72.

28. Polyak SJ, Khabar KS, Paschal DM, et al. Hepatitis C virus nonstructural 5A protein induces interleukin-8, leading to partial inhibition of the interferon-induced antiviral response. J Virol 2001; 75:6095-106.

29. Jia Y, Wei L, Jiang D, Wang J, Cong X, Fei R. Antiviral action of interferon-alpha against hepatitis $\mathrm{C}$ virus replicon and its modulation by interferon-gamma and interleukin-8. J Gastroenterol Hepatol 2007; 22:1278-85.

30. Zhang Y, Liu S, Wang H, et al. Elevated NIBP/TRAPPC9 mediates tumorigenesis of cancer cells through NFKB signaling. Oncotarget 2015; 6:6160-78.

31. Buster EH, Hansen BE, Lau GK, et al. Factors that predict response of patients with hepatitis B e antigen-positive chronic hepatitis B to peginterferon-alfa. Gastroenterology 2009; 137:2002-9.

32. Sonneveld MJ, Rijckborst V, Boucher CA, Hansen BE, Janssen HL. Prediction of sustained response to peginterferon alfa- $2 \mathrm{~b}$ for hepatitis $\mathrm{B}$ e antigen-positive chronic hepatitis B using on-treatment hepatitis B surface antigen decline. Hepatology 2010; 52:1251-7.

33. Sonneveld MJ, Brouwer WP, Janssen HL. Studies of IL28B genotype and response to peginterferon in chronic hepatitis B should be stratified by HBV genotype. Hepatology 2013; 57:1283. 